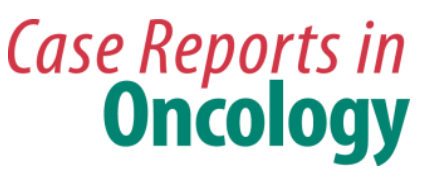

Case Rep Oncol 2018;11:239-245

DOI: $10.1159 / 000488388$

Published online: April 11, 2018

This article is licensed under the Creative Commons Attribution-NonCommercial 4.0 International License (CC BY-NC) (http://www.karger.com/Services/OpenAccessLicense). Usage and distribution for commercial purposes requires written permission.

\title{
Successful Clarithromycin Monotherapy in a Patient with Primary Follicular Lymphoma of the Duodenum
}

\author{
Barbara Kiesewetter $^{\mathrm{a}} \quad$ Werner Dolak $^{\mathrm{b}}$ Marius E. Mayerhoefer ${ }^{\mathrm{c}}$ \\ Ingrid Simonitsch-Klupp ${ }^{d}$ Markus Raderer ${ }^{a}$
}

${ }^{a}$ Clinical Division of Oncology, Department of Medicine I, Medical University of Vienna, Vienna, Austria; ${ }^{b}$ Clinical Division of Gastroenterology and Hepatology, Department of Medicine III, Medical University of Vienna, Vienna, Austria; 'Division of Nuclear Medicine, Department of Biomedical Imaging and Image-Guided Therapy, Medical University of Vienna, Vienna, Austria; dDepartment of Pathology, Medical University of Vienna, Vienna, Austria

\section{Keywords}

Primary follicular lymphoma of the duodenum · Extranodal lymphoma · Follicular lymphoma . Clarithromycin · Macrolides

\begin{abstract}
Primary follicular lymphoma of the duodenum (FL-D) constitutes a rare subtype of extranodal follicular lymphoma with a usually indolent course. To date, no distinct treatment recommendations have been defined for those patients. We report the case of a 58-year-old male patient presenting with endoscopically assessed, symptomatic FL-D who was treated with clarithromycin monotherapy in analogy to recent data for mucosa-associated lymphoid tissue lymphoma. Each treatment cycle consisted of clarithromycin $500 \mathrm{mg}$ twice daily for 3 weeks followed by a 2-week break. After four cycles of treatment, the patient showed a very good response with normal macroscopic findings confirmed by endosonographic examination and only focal minimal residual disease of lymphoma persisting in the histological assessment. The patient is currently asymptomatic and without treatment for $24+$ months. As clarithromycin combines antimicrobial and direct antiproliferative effects mediated through a
\end{abstract}


variety of pleiotropic mechanisms, this appears to be an interesting treatment approach for indolent lymphoma, particularly in those where a chronic infectious background cannot be completely ruled out, i.e., gastrointestinal manifestations. We suggest further investigation of this treatment approach.

\section{Introduction}

Primary intestinal follicular lymphoma of the duodenum (FL-D) is a rare type of extranodal follicular lymphoma and has been characterized as a rather indolent disease. Accounting for up to $40 \%$ of all primary duodenal lymphomas and approximately $1-4 \%$ of all gastrointestinal (GI)-lymphomas, it constitutes an important differential diagnosis for mucosaassociated lymphoid tissue lymphoma (MALT lymphoma) in case of gastroduodenal lesions $[1,2]$. Definition of FL-D requires exclusion of primary nodal lymphoma with secondary duodenal involvement, and is based on endoscopic assessment with multiple biopsies and endosonography followed by adequate immunohistochemical workup including demonstration of CD20-positive, CD10-positive, bcl2-positive, and CD5-negative lymphoid infiltrates [2-4].

Therapeutic approaches reported in the literature have usually been adapted from early-stage nodal follicular lymphoma and range from a wait-and-see policy to radiation, application of rituximab and anthracycline-based immunochemotherapy [2-4].

The macrolide-antibiotic clarithromycin has recently been studied for treatment of advanced or extragastric MALT lymphoma revealing surprisingly high response rates in heavily pretreated patients $[5,6]$. As this agent combines antimicrobial and direct immunoproliferative effects mediated through a variety of pleiotropic mechanisms, it appears to be an elegant treatment approach for indolent lymphomas, particularly when a chronic infectious/antigenic background is suspected, as has been exemplified by gastric MALT lymphoma and Helicobacter pylori (HP) infection [7].

In this paper, we report the first case of a patient with primary FL-D successfully being treated with clarithromycin monotherapy.

\section{Case Report}

In May 2014, a 58-year-old male patient was admitted to our department due to suspicion of FL-D. Initial reassessment of the external histology report confirmed diagnosis of FLD defined by adequate immunophenotyping of CD20+, CD79a+, bcl2+, CD10+, bcl6+, and CD3/CD5- and less than 5 centroblasts per high-power field (HPF) corresponding to lowgrade follicular lymphoma (G1). Endoscopy showed multiple nodules with superficial erosions in the post-bulbar region in an otherwise normal duodenal mucosa (Fig. 1). Further histologic assessment of gastric and esophagogastric tissue revealed mild chronic gastritis and esophagitis without lymphoma infiltration. No signs of infection with HP, i.e., neither on histology nor serology, were found in this patient, and no use of antibiotics within the last few years which might have resulted in inadvertent HP eradication was related by the patient.

Workup of medical records revealed a long-standing history of chronic dyspepsia, gastritis, and esophagitis including incipient Barrett syndrome aggravated by a hiatal hernia leading to laparoscopic fundoplication in January 2004. However, persistent episodes of 


\section{Case Reports in Oncology}

dyspepsia required repetitive proton pump inhibitor treatments and multiple endoscopic controls. Interestingly, the patient reported increasing abdominal discomfort in the last 6 months before the diagnosis of FL-D despite regular pantoprazole intake, which - in retrospect - was most probably related to lymphoma infiltration. Additional comorbidities consisted of status post hepatitis B (PCR negative) and moderate chronic obstructive pulmonary disease GOLD II.

When presenting at our clinic, the patient was in good health corresponding to an ECOG performance status of 0 and asymptomatic despite frequent abdominal discomfort as mentioned above. Primary laboratory workup showed regular blood counts as well as liver and kidney function parameters within normal limits. In analogy to MALT lymphoma, we screened the patient for potential autoimmune disorders including assessment of ANA/ ANCAs, thyroidal autoantibodies, rheumatoid factors, and SS-A/SS-B antibodies, which did not disclose clinically relevant findings. Serologic assessment of HP-specific IgG precluded chronic HP infection. For completion of primary lymphoma staging, an 18F-FDG-PET/CT was performed in June 2014, showing no lymphoma-related 18F-FDG uptake at the site of the lymphoma and no focal tracer accumulation in lymph nodes/further organs.

At the time of the second visit in the middle of June 2014, the patient was suffering from sinusitis and related ongoing abdominal pain, but was otherwise again in excellent condition. In view of the sinusitis plus our positive experience with clarithromycin for antiproliferative purpose in extranodal mucosal lymphomas, we decided to treat the patient with a long-term clarithromycin regimen as published before. Therapy consisted of clarithromycin $500 \mathrm{mg}$ twice daily for 3 weeks followed by a 2 -week break. As the first treatment cycle was well tolerated despite infrequent episodes of diarrhea grade I and slight dyseugesia grade I-II, a second cycle was subsequently given as planned.

In September 2014, the first restaging by upper GI endoscopy was performed and showed a significant decrease in size of lymphoma with only a single lesion being detectable (Fig. 2). Two further cycles of clarithromycin treatment were administered and again well tolerated. At the next visit in April 2015, the patient reported a clear reduction of GI symptoms, i.e., disappearance of abdominal pain and dyspepsia. He was consequently able to discontinue proton pump inhibitor treatment with pantoprazole. Re-endoscopy revealed regular appearance of the duodenal mucosa and only minimal focal remnants of lymphoma were detected by histology (Fig. 3). Treatment with clarithromycin was thus terminated and a wait-and-see approach was chosen.

The next restaging was performed in October 2015 and demonstrated absence of any lymphomatous lesions upon endoscopy, and also no lymphoma remnants were detected on histological assessment of biopsy specimens. However, due to the normal macroscopic findings, the number of biopsies was smaller than during the initial examinations and consisted of only 5 biopsies. The next follow-up examination was performed in March 2016, and showed macroscopic remission both on conventional endoscopy as well as endosonographic assessment. Histological workup revealed focal persistence of lymphoma in 2 out of 10 biopsies corresponding to minimal residual disease. In view of this, the complete remission seen in the prior endoscopy was interpreted as a sampling error due to the low number of biopsies in the presence of only scattered microscopic lymphoma foci. The patient was further followed by endoscopy every 4-6 months and has not shown any signs of symptomatic or histological progression until today.

Taken together, our patient achieved an ongoing remission with four cycles of clarithromycin monotherapy and is asymptomatic and free of progress after a current followup time of $24+$ months after initiation of clarithromycin. 
 Oncology}

\section{Discussion}

Clarithromycin is a macrolide antibiotic, which displays not only antimicrobial effects but also pleiotropic immunomodulatory and antineoplastic properties. In fact, it appears to enhance antitumor activity of macrophages, to inhibit tumor necrosis factor alpha (TNF- $\alpha$ ) and vascular endothelial growth factor (VEGF) levels, to reduce the neutrophil production of interleukin-6 (IL-6), and to induce direct apoptotic changes in tumor cells as underlined by in vivo and ex vivo data $[5,6,8]$. As a proof of principle, clarithromycin has been used as a "re-sensitizer" in patients with multiple myeloma refractory to therapy with lenalidomide and dexamethasone, but has also been used upfront both in combination with thalidomide/ dexamethasone (BiTD) and lenalidomide/dexamethasone (BiRD) with an objective response rate of up to $90 \%$, and effectively almost doubling expected responses with the respective two drug regimens [9]. In addition to these data, two phase II trials on clarithromycin monotherapy for heavily pretreated gastric and extragastric MALT lymphoma patients have been published, suggesting promising activity with a response rate of $52 \%(95 \%$ CI 32-72) in a recently published multicenter study $[5,6]$.

The decision to treat the current patient with clarithromycin was based on the promising trials for other mucosa-restricted lymphomas as MALT lymphomas, the fact that due to available data first-line treatment of FL-D should be minimally toxic, and the indolent course of FL-D, which does not necessarily require fast-acting therapy. In terms of pathological features, FL-D represents a very distinct type of low-grade lymphoma underlined by the observation that the histologically assessed grading was 1 or 2 throughout published data [1-4] with virtual absence of more aggressive histologies. In addition, it appears that $t(14 ; 18)$ (q32;q21)-positive FL-D does not develop any additional karyotypic changes, which further supports the particularly benign character of this disease with minimal risk of developing more aggressive lymphoma in the course of the disease [3]. The typical clinical findings of FL-D include unspecific abdominal discomfort and endoscopically assessed multiple polypoid lesions in the duodenum, while in contrast MALT lymphoma presents with rather diffuse infiltrates or erosions $[2-4,10]$. These polypoid lesions, however, have been reported to accumulate around/opposite the papilla, which may be suggestive of an antigenic/infectious drive excreted via the biliary tract and constitute a further hypothetical similarity to MALT lymphoma of such a "mucosa-associated follicular lymphoma."

Similar to the treatment recommendations for primary nodal follicular lymphoma, patients with FL-D reported in the literature have been successfully approached with rituximab monotherapy, radiotherapy, or chemotherapy-based regimens in the past [2-4]. However, a wait-and-see policy in referral to asymptomatic, nonprogressive nodal follicular lymphoma appears feasible as no transformation or lymphoma-related death has been observed in the largest collective of FL-D published so far following 63 patients for an impressive median time of 77 months (range 12-177) [3]. In this series, patients from several German and Austrian centers with different individual treatment approaches were analyzed and particularly the wait-and-see cohort revealed surprising observations: out of 24 patients without specific treatment for lymphoma, a total of 7 experienced a complete remission during follow-up and an additional 6 showed signs of spontaneous regression. None of these 24 patients progressed during the observation period. Patients treated with radiotherapy or chemotherapy responded well to treatment, but in 3 of 8 patients receiving chemotherapy a local relapse was noticed.

While no formal guidelines for the treatment of FL-D have been established, consensus between the two largest retrospective series $[3,4]$ favors either wait and see or radiothera- 
py. However, additional forms of therapy are still warranted, as radiotherapy may still result in abdominal complaints/side effects, while the wait-and-see approach might put some emotional stress on the patient, even if data for the indolent clinical course appear solid. In addition, a small percentage of patients are symptomatic (as was the case in our patient) and require therapy for relief of symptoms.

As has been stated above, also the antimicrobial effects of clarithromycin have to be taken into consideration for the treatment response observed in our patient in view of a potential - but as yet undefined - infectious cause of FL-D. Despite the fact that this patient was shown to be HP negative, there are data in the literature reporting an association also for non-MALT GI lymphoma and infectious agents, i.e., HP for primary gastric diffuse large B-cell lymphoma or Campylobacter jejuni for immunoproliferative intestinal disease [11, 12]. Corresponding eradication treatment was an effective way to manage these patients in some cases. It has been speculated that FL-D might have features intermediate between nodal follicular lymphoma and MALT lymphoma due to, e.g., aberrant VH gene types and potential expression of $\alpha-4 / \beta$-7-integrins on FL-D infiltrates, which are also typical for MALT lymphoma cells $[13,14]$. Toyoda et al. [15] have reported a single case of complete remission of FL-D following HP eradication. However, to date the potential role of infectious causes has not been systematically evaluated in FL-D, and the larger retrospective series, which have included patients who had undergone HP eradication, so far could not substantiate a role for this form of therapy in FL-D [2-4].

To the best of our knowledge, we report the first case of an ongoing remission in FL-D following monotherapy with clarithromycin. While currently no accurate explanation for the mode of action of clarithromycin in this distinct entity may be offered, we believe that further evaluation of this treatment concept appears highly interesting in this indolent disease based on promising data in extranodal lymphoma of MALT.

\section{Statement of Ethics}

Informed consent was obtained from the patient for publication of this case report.

\section{Disclosure Statement}

The authors certify that they have no conflicts of interest to disclose in the subject matter discussed in this paper.

\section{References}

1 Yoshino T, Miyake K, Ichimura K, Mannami T, Ohara N, Hamazaki S et al. Increased incidence of follicular lymphoma in the duodenum. Am J Surg Pathol. 2000 May;24(5):688-93.

2 Yamamoto S, Nakase H, Yamashita K, Matsuura M, Takada M, Kawanami C et al. Gastrointestinal follicular lymphoma: review of the literature. J Gastroenterol. 2010 Apr;45(4):370-88.

3 Schmatz AI, Streubel B, Kretschmer-Chott E, Püspök A, Jäger U, Mannhalter C et al. Primary follicular lymphoma of the duodenum is a distinct mucosal/submucosal variant of follicular lymphoma: a retrospective study of 63 cases. J Clin Oncol. 2011 Apr;29(11):1445-51.

4 Damaj G, Verkarre V, Delmer A, Solal-Celigny P, Yakoub-Agha I, Cellier C et al. Primary follicular lymphoma of the gastrointestinal tract: a study of 25 cases and a literature review. Ann Oncol. 2003 Apr;14(4):623-9. 
 Oncology}

\begin{tabular}{l|l}
\hline Case Rep Oncol 2018;11:239-245 \\
\hline DOI: 10.1159/000488388 & $\begin{array}{l}\text { ( ) 2018 The Author(s). Published by S. Karger AG, Basel } \\
\text { www.karger.com/cro }\end{array}$ \\
\hline
\end{tabular}

Kiesewetter et al.: Successful Clarithromycin Monotherapy in a Patient with Primary Follicular Lymphoma of the Duodenum

5 Govi S, Dognini GP, Licata G, Crocchiolo R, Resti AG, Ponzoni M et al. Six-month oral clarithromycin regimen is safe and active in extranodal marginal zone B-cell lymphomas: final results of a single-centre phase II trial. Br J Haematol. 2010 Jul;150(2):226-9.

6 Ferreri AJ, Sassone M, Kiesewetter B, Govi S, Scarfò L, Donadoni G et al. High-dose clarithromycin is an active monotherapy for patients with relapsed/refractory extranodal marginal zone lymphoma of mucosaassociated lymphoid tissue (MALT): the HD-K phase II trial. Ann Oncol. 2015 Aug;26(8):1760-5.

7 Raderer M, Kiesewetter B, Ferreri AJ. Clinicopathologic characteristics and treatment of marginal zone lymphoma of mucosa-associated lymphoid tissue (MALT lymphoma). CA Cancer J Clin. 2016 MarApr;66(2):153-71.

8 Ferreri AJ. Activity of clarithromycin in mucosa-associated lymphoid tissue-type lymphomas: antiproliferative drug or simple antibiotic? Chest. 2011 Mar;139(3):724-5.

9 Mark TM, Coleman M. It's time to take clarithromycin seriously in multiple myeloma. Acta Haematol. 2016;135(2):101-2.

10 Iwamuro M, Kondo E, Takata K, Yoshino T, Okada H. Diagnosis of follicular lymphoma of the gastrointestinal tract: A better initial diagnostic workup. World J Gastroenterol. 2016 Jan;22(4):1674-83.

11 Kuo SH, Yeh KH, Wu MS, Lin CW, Hsu PN, Wang HP et al. Helicobacter pylori eradication therapy is effective in the treatment of early-stage $\mathrm{H}$ pylori-positive gastric diffuse large B-cell lymphomas. Blood. 2012 May;119(21):4838-44.

12 Lecuit M, Abachin E, Martin A, Poyart C, Pochart P, Suarez F et al. Immunoproliferative small intestinal disease associated with Campylobacter jejuni. N Engl J Med. 2004 Jan;350(3):239-48.

13 Sato Y, Ichimura K, Tanaka T, Takata K, Morito T, Sato $\mathrm{H}$ et al. Duodenal follicular lymphomas share common characteristics with mucosa-associated lymphoid tissue lymphomas. J Clin Pathol. 2008 Mar;61(3):377-81.

14 Bende RJ, Smit LA, Bossenbroek JG, Aarts WM, Spaargaren M, de Leval L et al. Primary follicular lymphoma of the small intestine: alpha4beta7 expression and immunoglobulin configuration suggest an origin from local antigen-experienced B cells. Am J Pathol. 2003 Jan;162(1):105-13.

15 Toyoda H, Yamaguchi M, Nakamura S, Nakamura T, Kimura M, Suzuki H et al. Regression of primary lymphoma of the ampulla of Vater after eradication of Helicobacter pylori. Gastrointest Endosc. 2001 Jul;54(1):92-6.

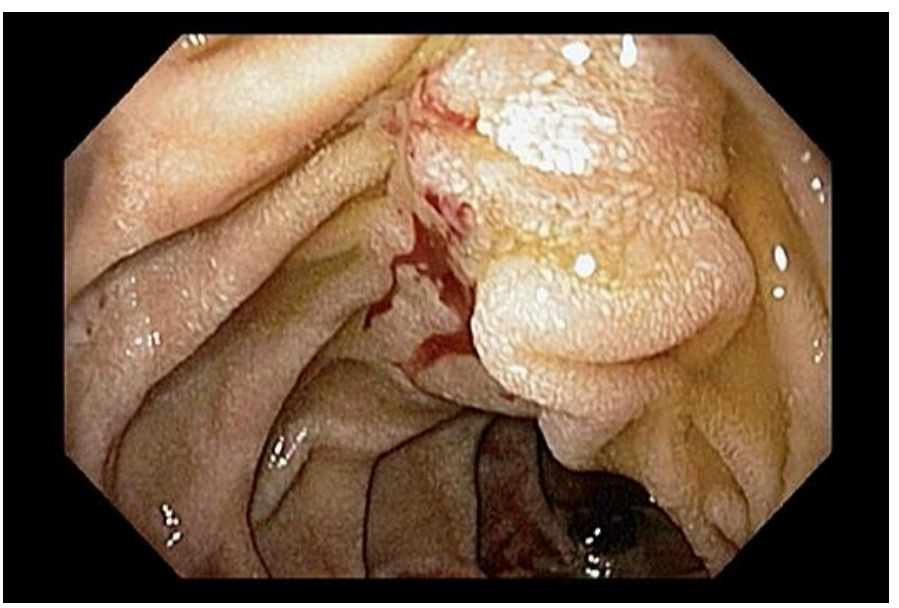

Fig. 1. Endoscopic picture of the second part of the duodenum showing a nodular mass with superficial erosion and active bleeding. 


\section{Case Reports in Oncology}

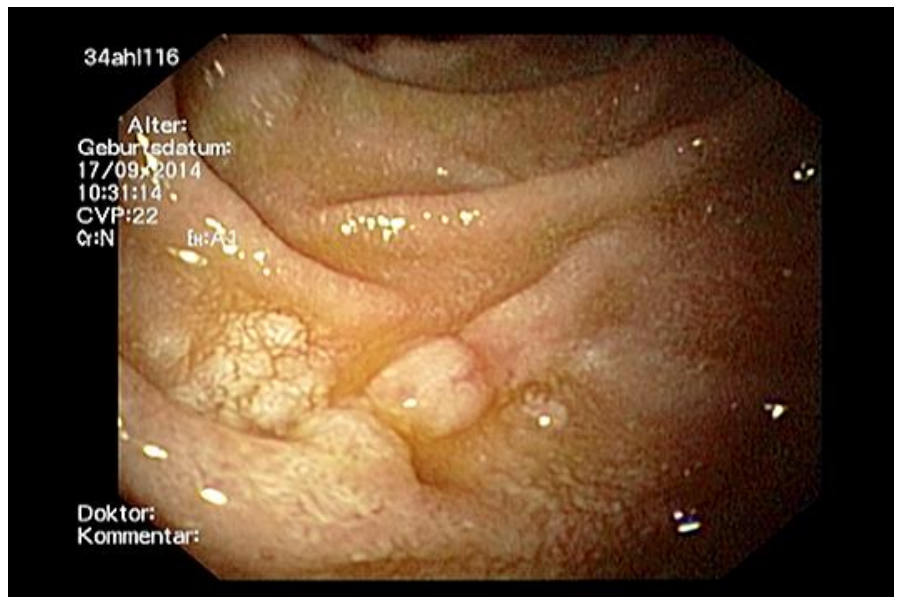

Fig. 2. Endoscopic picture of the second part of the duodenum showing small nodules with focal erosion.

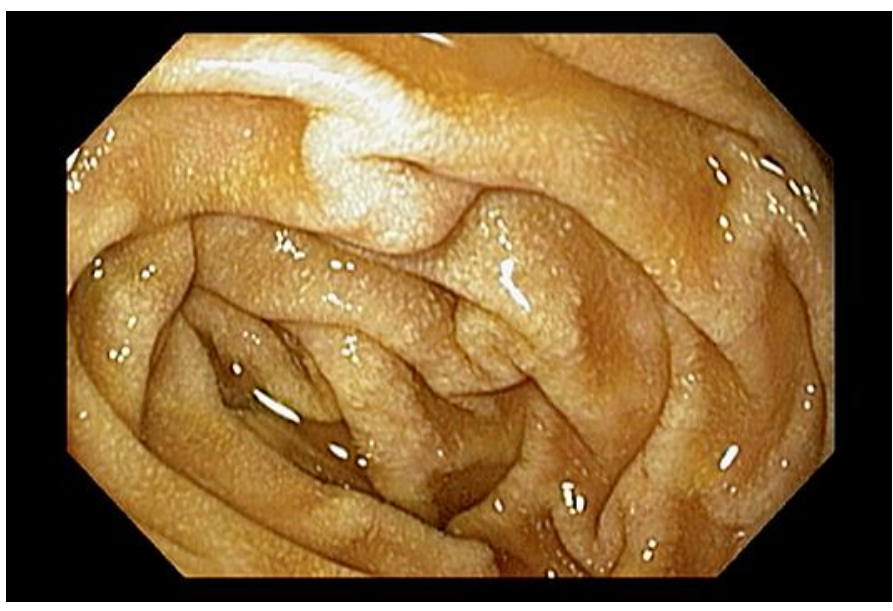

Fig. 3. Normal endoscopic picture of the second part of the duodenum. 